\title{
TeV-scale Majorogenesis
}

\author{
Yoshihiko Abe, Yu Hamada, Takahiro Ohata, Kenta Suzuki and Koichi Yoshioka \\ Department of Physics, Kyoto University, \\ Kyoto 606-8502, Japan \\ E-mail: y.abe@gauge.scphys.kyoto-u.ac.jp, \\ yu.hamada@gauge.scphys.kyoto-u.ac.jp, \\ tk. ohata@gauge.scphys.kyoto-u.ac.jp, \\ S.Kenta@gauge.scphys.kyoto-u.ac.jp, \\ yoshioka@gauge.scphys.kyoto-u.ac.jp
}

AbSTRACT: The Majoron, the Nambu-Goldstone boson of lepton number symmetry, is an interesting candidate for dark matter as it deeply connects the dark matter and neutrino physics. In this paper, we consider the Majoron dark matter as pseudo Nambu-Goldstone boson with $\mathrm{TeV}$-scale mass. The heavy Majoron generally has the large decay constant and tiny Yukawa couplings to light right-handed neutrinos which are required by cosmological and astrophysical observations. That makes it difficult to realize the desired amount of the relic abundance of Majoron dark matter. We consider three improved scenarios for the generation of Majoron, dubbed as Majorogenesis, in the early universe and find in all cases the parameter space compatible with the relic abundance and cosmic-ray constraints.

Keywords: Beyond Standard Model, Cosmology of Theories beyond the SM, Neutrino Physics

ARXiv EPrint: 2004.00599 


\section{Contents}

1 Introduction $\quad 1$

2 Majoron dark matter 2

2.1 The model 2

2.2 Decaying dark matter 3

3 Dark matter creation: Majorogenesis 5

3.1 Flaw and improvements of the model 5

3.2 (A): heavy RH neutrino decay $\quad 6$

3.3 (B): scalar portal interaction 9

3.4 (C): resonant creation from non-thermal source 12

4 Summary and outlook 14

\section{Introduction}

The existence of dark matter (DM) is clear from various observations over the past decades, such as galaxy rotation curves [1,2], gravitational lensing [3], cosmic microwave background [4] and collision of Bullet Cluster [5]. While there are various constraints on the DM mass and the scattering cross section from astrophysical observations and direct detection experiments [6-10], the nature of DM is still unknown. The identification of DM is important not only for cosmology but also for particle physics, because there are no particle contents playing the role of DM in the Standard Model(SM). DM would be the key to investigate new physics beyond the SM.

Another important issue that is unanswered by the SM is the neutrino masses [11], which is implied by the observations of neutrino oscillation. One way to realize the tiny neutrino mass scale is the see-saw mechanism [12-14]. In the type-I see-saw, right-handed(RH) neutrinos and their Majorana masses are introduced to obtain the realistic neutrino masses. However, the origin of the Majorana masses is not explained within the model. In the socalled Majoron model [15-17], a new SM-singlet complex scalar is introduced to explain it. The scalar develops a vacuum expectation value(VEV) breaking a global U(1) symmetry, which provides the Majorana masses as the SM Higgs mechanism. Corresponding to the symmetry breaking, there arises a pseudo scalar particle called the Majoron, which is the Nambu-Goldstone boson(NGB) associated with the U(1) symmetry. In refs. [18-23], it is discussed that the Majoron can be a DM candidate by introducing explicit breaking terms for the U(1) symmetry. In particular, the Majoron becomes a pseudo NGB(pNGB) when the soft breaking mass term is introduced as in ref. [18]. A remarkable feature of pNGB DM 
is the derivative coupling with other (scalar) particles which enables us to avoid the constraints from the direct detection experiments [24-27]. For further studies on the Majoron DM and the pNGB DM, see, e.g., refs. [28-36]. The origin of the U(1) breaking term in the scalar potential is discussed in various contexts such as the effect of quantum gravity [37], neutrino Dirac Yukawa coupling [38], coupling with another scalar [39, 40] and so on.

On the other hand, some cosmic-ray observations are known to suggest the existence of leptophilic TeV-scale DM. That motivates us to consider a TeV-scale Majoron DM, whose mass scale is heavier than those in previous works. The heavy Majoron can decay to neutrinos, which requires the SM singlet scalar VEV is around the unification scale. It can also decay to heavy quarks such as the top quark, and that imposes strong upper bound on the Yukawa couplings between the Majoron and the RH neutrinos. The Majoron interactions are too small to realize the DM relic abundance via the thermal freeze-out mechanism [36]. Hence the creation of Majoron DM (dubbed as Majorogenesis) at $\mathrm{TeV}$ scale should be realized in a way other than the freeze-out mechanism, such as the freeze-in production [41].

In this paper, we investigate the Majorogenesis for TeV-scale Majoron. We then consider the following three scenarios; (A) introducing explicit Majoron masses, (B) using the interaction with the SM Higgs doublet $(\mathrm{C})$ using the resonant production from non-thermal $\mathrm{RH}$ neutrinos. All of these scenarios are found to have the parameter space compatible with the tiny Yukawa coupling and the DM relic abundance.

This paper is organized as follows. In section 2, we discuss the Majoron model and its phenomenological constraints from heavy Majoron DM decays. In section 3, we show the difficulty of creating the heavy Majoron in the reference model, and then consider three ways to realize the $\mathrm{TeV}$-scale Majorogenesis. In each case, we will evaluate the Majoron relic abundance and show the parameter space realizing the TeV-scale Majorogenesis. Section 4 is devoted to summarizing our results and discussing future work.

\section{Majoron dark matter}

\section{$2.1 \quad$ The model}

First of all, we consider the reference Majoron model for the following discussion. We introduce a new SM-singlet complex scalar which has the Yukawa coupling to RH neutrinos. The Lagrangian for the $\mathrm{RH}$ neutrinos $\nu_{R i}$ are written as

$$
\mathcal{L}_{N}=i \overline{\nu_{R i}} \not \partial \nu_{R i}-\frac{f_{i j}}{2} \Phi \overline{\nu_{R i}^{c}} \nu_{R j}-y_{\alpha i}^{\nu} \overline{L_{\alpha}} \tilde{H} \nu_{R i}+\text { h.c. },
$$

where the RH neutrinos and the new scalar $\Phi$ have the lepton number +1 and -2 , respectively. The neutrino Yukawa coupling $y_{\alpha i}^{\nu}$ gives the Dirac mass $m_{D}=y^{\nu} v / \sqrt{2}$ after the electroweak symmetry breaking, where $v$ is the electroweak VEV $v \simeq 246 \mathrm{GeV}$. In addition, the new Yukawa coupling with $\Phi$ gives the Majorana mass $M_{N}=f v_{\phi} / \sqrt{2}$. Thus, the small masses for active neutrinos are generated by the type-I seesaw mechanism as $\left(m_{\nu}\right)_{\alpha \beta} \approx-\left(m_{D}\right)_{\alpha i}\left(M_{N}^{-1}\right)_{i j}\left(m_{D}^{\mathrm{T}}\right)_{j \beta}$. We use Geek indices $\alpha, \beta, \ldots$ for the generation of the SM leptons and Latin indices $i, j, \ldots$ for the generation of the $\mathrm{RH}$ neutrinos. 
The scalar potential in the model is written as

$$
V(H, \Phi)=V_{H}(H)-\frac{\mu_{\Phi}^{2}}{2}|\Phi|^{2}+\frac{\lambda_{\Phi}}{2}|\Phi|^{4}-\frac{m^{2}}{4}\left(\Phi^{2}+\Phi^{* 2}\right),
$$

where $V_{H}$ is the Higgs potential in the SM and the coupling between $\Phi$ and $H$ will be taken into account in section 3.3. The last quadratic term proportional to $m^{2}$ is the soft-breaking term to generate the pNGB mass. This term breaks the $\mathrm{U}(1)_{L}$ symmetry of the scalar potential into $\mathbb{Z}_{2}$, which corresponds to $\Phi \rightarrow-\Phi .{ }^{1}$ For the potential stability, the quartic coupling satisfies $\lambda_{\Phi}>0$. The scalar field develops a VEV $v_{\phi}$, and is parametrized as

$$
\Phi=\frac{v_{\phi}+\phi+i \chi}{\sqrt{2}}
$$

The stationary conditions are solved as $\mu_{\Phi}^{2}=\lambda_{\Phi} v_{\phi}^{2}-m^{2}$, and the scalar masses in the $\mathrm{U}(1)_{L}$ breaking vacuum are given by

$$
m_{\phi}^{2}=\lambda_{\Phi} v_{\phi}^{2}, \quad m_{\chi}^{2}=m^{2} .
$$

The CP-odd component $\chi$ is a pNGB called as the Majoron, whose mass is given by the soft-breaking parameter $m$. In the following parts of this paper, we will see that this Majoron can be a DM candidate.

In general, the Yukawa matrix $f_{i j}$ in eq. (2.1) can be diagonalized into $f_{i} \delta_{i j}$ by the redefinition of the $\mathrm{RH}$ neutrinos and the diagonal couplings $f_{i}$ are taken to be real. The Majorana fermion in this mass basis is denoted by $N_{i}=\nu_{R i}+\nu_{R i}^{c}$, in which we denote the redefined RH neutrino as $\nu_{R i}$. The Lagrangian is rewritten using these Majorana fermions as

$$
\begin{aligned}
\mathcal{L}_{N}= & \frac{i}{2} \overline{N_{i}} \not \partial N_{i}-\frac{M_{N_{i}}}{2} \overline{N_{i}} N_{i}-\frac{f_{i}}{2 \sqrt{2}} \phi \overline{N_{i}} N_{i}-\frac{i f_{i}}{2 \sqrt{2}} \chi \overline{N_{i}} \gamma_{5} N_{i} \\
& -Y_{\alpha i}^{\nu} \overline{L_{\alpha}} \tilde{H} P_{R} N_{i}+\text { h.c. },
\end{aligned}
$$

where $Y_{\alpha i}^{\nu}$ is the neutrino Yukawa matrix in the $\mathrm{RH}$ neutrino mass basis and $P_{R / L}$ is the chirality projection. An important point is that the flavor changing off-diagonal interaction between the Majoron and the RH neutrinos such as $\chi \overline{N_{1}} N_{2}$ disappears in the mass diagonal basis.

\subsection{Decaying dark matter}

In this subsection, we see features of the TeV-scale Majoron and the phenomenological constraints as the DM candidate. The Majoron is assumed to be lighter than the lightest $\mathrm{RH}$ neutrino to prevent it from decaying into the $\mathrm{RH}$ neutrinos. Otherwise, the Yukawa coupling $f$ is required to be highly suppressed and/or the VEV $v_{\phi}$ must be huge due to astrophysical constraints.

\footnotetext{
${ }^{1}$ The total Lagrangian with this soft-breaking term is invariant under the $\mathbb{Z}_{4}$ symmetry, which is the residual discrete symmetry of the global $\mathrm{U}(1)_{L}$.
} 

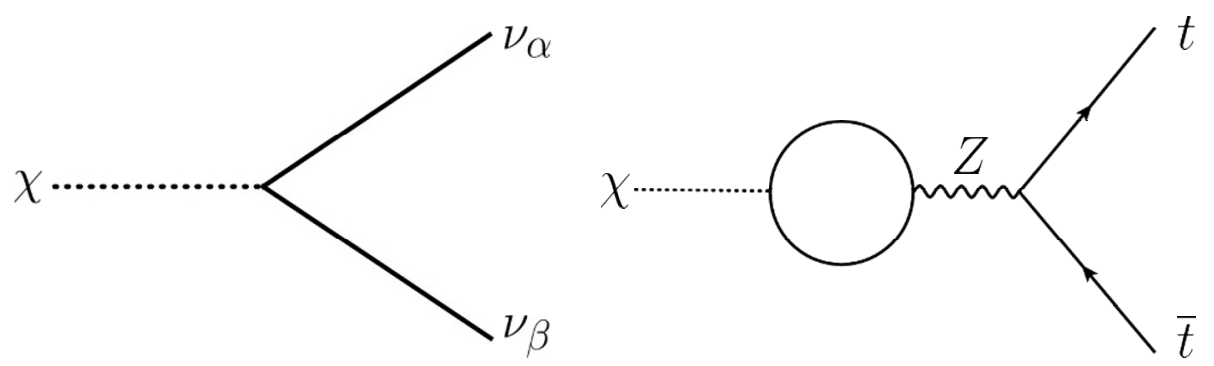

Figure 1. The Feynman diagrams for the DM decay processes. (left): $\chi \rightarrow \nu_{\alpha} \nu_{\beta}$. (right): $\chi \rightarrow t \bar{t}$. The internal lines in the loop represent the active and heavy neutrinos.

The massive Majoron is unstable due to its interaction with $\mathrm{RH}$ neutrinos and the neutrino Yukawa couplings. The main decay channels are expressed by the Feynman diagrams of figure 1 . The decay width to the neutrinos is given by

$$
\Gamma_{\chi \rightarrow \nu_{\alpha} \nu_{\beta}}=\frac{m_{\chi}}{16 \pi v_{\phi}^{2}}\left|\left(m_{\nu}\right)_{\alpha \beta}\right|^{2}
$$

where $\left(m_{\nu}\right)_{\alpha \beta}$ is the neutrino mass matrix. To realize the long-lived DM, the VEV $v_{\phi}$ has a lower bound for a fixed value of the DM mass $m_{\chi}$. The constraints on the DM mass and lifetime for this decay mode are discussed e.g., in refs. [28, 29]. For example, the VEV $v_{\phi}$ is found to satisfy $v_{\phi} \gtrsim 10^{15} \mathrm{GeV}$ for $\mathrm{TeV}$-scale DM. In the following parts, we assume $v_{\phi} \approx 10^{15} \mathrm{GeV}$. In addition, the Majoron is so heavy that it can decay to (the top) quark pair through the one-loop diagram shown figure 1. As the width is generally proportional to the quark mass, the dominant radiative decay is given by $\chi \rightarrow t \bar{t}$, if possible, and its width is evaluated as

$$
\Gamma_{\chi \rightarrow t \bar{t}}=\frac{3 \alpha_{W} C_{\chi Z}^{2}}{8 \cos ^{2} \theta_{W}} \frac{m_{\chi} m_{t}^{2}}{m_{Z}^{4}} \sqrt{1-\frac{4 m_{t}^{2}}{m_{\chi}^{2}}}
$$

where $m_{t}$ and $m_{Z}$ are the masses of the top quark and the $Z$ boson, respectively, and $\alpha_{W}$ is the fine structure constant of $\mathrm{SU}(2)_{L}$ gauge coupling. The overall factor 3 comes from the summation of color indices of the final states. The neutrino loop factor connecting $\chi$ and $Z$ is given by

$$
C_{\chi Z}=\sum_{i, j} \frac{g\left|\left(m_{D}\right)_{i j}\right|^{2}}{16 \pi^{2} v_{\phi} \cos \theta_{W}} \int_{0}^{1} d x \int_{0}^{1-x} d y \int_{0}^{1-x-y} d z \frac{F\left(2 m_{\chi}^{2} / M_{N_{i}}^{2}\right)}{F\left(m_{\chi}^{2} / M_{N_{i}}^{2}\right)^{2}},
$$

where $F(\omega) \equiv(x+y)+(y+z)(y+z-1) \omega$. The main decay modes of the Majoron are these $\chi \rightarrow \nu_{\alpha} \nu_{\beta}, \chi \rightarrow t \bar{t}$, and the model parameters are constrained by the cosmic-ray observations such as anti-protons and gamma-rays. The decay widths of the Majoron to other SM particles are much smaller, then the constraints are irrelevant. ${ }^{2}$ In general, analyzing the constraints on the model parameters are very complicated due to many degrees of freedom

\footnotetext{
${ }^{2}$ In the case of the Majoron being light, see a previous work [42] for the constraints from the Majoron decay.
} 
and indeterminacy [43], which is beyond the scope of this paper. In this paper, we impose a conservative upper bound on the Yukawa coupling $f_{i} \lesssim 10^{-(10-11)}$ with reference to the past analysis, but the precise value of $f_{i}$ is irrelevant to the Majoron creation.

From these results and analysis, we find the following three statements are inseparable in the TeV-scale Majoron DM model:

1. Light $\mathrm{RH}$ neutrinos with $\mathrm{TeV}-\mathrm{PeV}$-scale masses

2. Heavy Majoron feebly interacting with RH neutrinos

3. Large VEV of $\Phi$ around the unification scale

\section{Dark matter creation: Majorogenesis}

In this section, we will show the difficulty to realize the DM relic abundance, and discuss some improved scenarios for the Majorogenesis to take place.

\subsection{Flaw and improvements of the model}

As we have seen in the last section, the three conditions, 1. light RH neutrinos, 2. heavy Majoron, 3. large $v_{\phi}$, are inseparable when we consider a TeV-scale Majoron DM. In the model in section 2, the Majoron couples to the SM particles only through the RH neutrinos and the coupling is too small to realize the freeze-out mechanism. Even if we introduce the mixing coupling such as $\lambda_{H \Phi}|H|^{2}|\Phi|^{2}$, it is hard for pNGB DM with the large VEV to realize the relic abundance as by the freeze-out mechanism [36]. Then another option to create the Majoron is the freeze-in mechanism discussed in ref. [41]. The magnitude of the coupling that is necessary for the freeze-in to work is typically $\mathcal{O}\left(10^{-11}\right)$, and thus the tiny Yukawa couplings in the model of section $2, f_{i j} \lesssim \mathcal{O}\left(10^{-(10-11)}\right)$, seem useful for the Majoron creation via the freeze-in. However, the Yukawa interaction between the Majoron and the RH neutrinos is flavor diagonal in the RH neutrino mass basis, and flavor changing off-diagonal interactions such as $\chi \overline{N_{1}} N_{2}$ are absent in the Lagrangian (see eq. (2.5)). The other processes are too tiny to explain the relic abundance by the freeze-in mechanism. The scattering amplitude of the annihilation $N N \rightarrow \chi \chi$ via $t$-channel is proportional to $f^{2}$. In addition, the decay $N \rightarrow \chi \nu$ is highly suppressed by the neutrino mass on top of $f$. Therefore, it is impossible to realize the DM relic abundance by the freeze-in mechanism using the RH neutrino decay in that model. Here let us consider the following three scenarios to avoid this flaw.

(A). The first is to modify the universality of mass/coupling ratios for the pNGB Majoron. A simple way for this is to introduce Majorana masses for $\mathrm{RH}$ neutrinos, which break the $\mathrm{U}(1)_{L}$ symmetry similarly to the soft breaking term for $\Phi$. Then flavor changing couplings of the Majoron generally appear in the RH neutrino mass basis, and could lead to the freeze-in production of Majoron. 


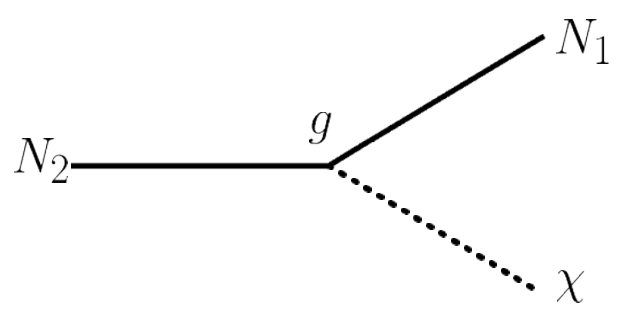

Figure 2. The Feynman diagram for Majorogenesis in the scenario (A).

(B). The second is adding the mixing coupling between the SM Higgs $H$ and the SM singlet scalar $\Phi$ such as $\lambda_{H \Phi}|H|^{2}|\Phi|^{2}$ to the scalar potential (2.2). The Majoron can interact with the SM Higgs via this coupling on top of neutrinos, but the typical magnitude of the interaction is also too small to realize the thermal relic because of the nature of NGB [35] as we stated above. As an alternative option, we consider the freeze-in mechanism through this portal coupling.

(C). The third option is using a non-thermal creation of the $\mathrm{RH}$ neutrinos during the reheating after the cosmological inflation. The scattering process mediated by the CP-even scalar particle arising from $\Phi$ is essential to explain the DM relic abundance.

In the rest part of this section, we discuss the above three scenarios $(\mathrm{A})-(\mathrm{C})$ and investigate the parameter space realizing the $\mathrm{TeV}$-scale Majorogenesis for each case.

\section{2 (A): heavy RH neutrino decay}

Let us consider the scenario (A), in which Majorana mass terms for the RH neutrinos are introduced:

$$
\Delta \mathcal{L}_{\text {Majorana }}=-\frac{1}{2} m_{i j} \overline{\nu_{R i}^{c}} \nu_{R j}+\text { h.c. }
$$

which enables the flavor changing interactions in the mass-diagonal basis. In this subsection, we consider only two RH neutrinos $(i=1,2)$, or equivalently, we assume that one of the three is sufficiently heavy. Hereafter, we use $g$ for the off-diagonal Yukawa interaction giving $\chi \overline{N_{1}} N_{2}$ vertex, which is assumed to have the constraint,

$$
g \lesssim 10^{-10}
$$

as in the Majoron model.

The DM creation process is $N_{2} \stackrel{g}{\rightarrow} N_{1} \chi\left(M_{N_{2}}>M_{N_{1}}+m_{\chi}\right)$, which is shown in figure 2, and the decay width is given by

$$
\Gamma_{N_{2} \rightarrow N_{1} \chi}=\frac{g^{2} M_{N_{2}}}{32 \pi} I\left(\frac{M_{N_{1}}}{M_{N_{2}}}, \frac{m_{\chi}}{M_{N_{2}}}\right)
$$

where the function $I(x, y)$ is defined by $I(x, y) \equiv\left[(1-x)^{2}-y^{2}\right]^{3 / 2}\left[(1+x)^{2}-y^{2}\right]^{1 / 2}$. On the other hand, the thermal creation process of the $\mathrm{RH}$ neutrinos are given by $N_{i} \longleftrightarrow$ $L_{\alpha} H\left(L_{\alpha}^{c} H^{\dagger}\right)$, and the decay width is expressed as

$$
\Gamma_{N_{i} \rightarrow \mathrm{B}}=\frac{\left|Y_{\alpha i}^{\nu}\right|^{2} M_{N_{i}}}{8 \pi} .
$$


The Boltzmann equations for the RH neutrinos and the Majoron are given by

$$
\begin{aligned}
\frac{d Y_{N_{2}}(x)}{d x}= & -\frac{\Gamma_{N_{2} \rightarrow N_{1} \chi}}{H x} \frac{K_{1}\left(r_{2} x\right)}{K_{2}\left(r_{2} x\right)} Y_{N_{2}}(x) \\
& -\frac{\Gamma_{N_{2} \rightarrow \mathrm{B}}}{H x} \frac{K_{1}\left(r_{2} x\right)}{K_{2}\left(r_{2} x\right)}\left[Y_{N_{2}}(x)-Y_{N_{2}}^{\mathrm{eq}}\left(r_{2} x\right)\right], \\
\frac{d Y_{N_{1}}(x)}{d x}= & +\frac{\Gamma_{N_{2} \rightarrow N_{1} \chi}}{H x} \frac{K_{1}\left(r_{2} x\right)}{K_{2}\left(r_{2} x\right)} Y_{N_{2}}(x) \\
& -\frac{\Gamma_{N_{1} \rightarrow \mathrm{B}}}{H x} \frac{K_{1}\left(r_{1} x\right)}{K_{2}\left(r_{1} x\right)}\left[Y_{N_{1}}(x)-Y_{N_{1}}^{\mathrm{eq}}\left(r_{1} x\right)\right], \\
\frac{d Y_{\chi}(x)}{d x}= & +\frac{\Gamma_{N_{2} \rightarrow N_{1} \chi}}{H x} \frac{K_{1}\left(r_{2} x\right)}{K_{2}\left(r_{2} x\right)} Y_{N_{2}}(x),
\end{aligned}
$$

where $H$ denotes the Hubble parameter, $K_{n}$ is the modified Bessel function of the second kind. We introduce the dimensionless parameter $x$ by $x \equiv m_{\chi} / T$ for the temperature $T$ and the mass ratios by $r_{i} \equiv M_{N_{i}} / m_{\chi}$. The yield of a particle $X$ is defined by $Y_{X} \equiv$ $n_{X} / s$ with $n_{X}$ and $s$ being the number density of $X$ and the entropy density, respectively. The temperature dependence of the Hubble parameter and the entropy density is $H=$ $\sqrt{4 \pi^{3} g_{*} / 45} T^{2} / m_{\mathrm{Pl}}$ and $s=2 \pi^{2} g_{*}^{S} T^{3} / 45$ with the Planck mass $m_{\mathrm{Pl}}$. The function form of $Y_{X}$ in the thermal equilibrium is given by

$$
Y_{X}^{\mathrm{eq}}(z)=g_{X}\left(\frac{45}{4 \pi^{4} g_{*}^{S}}\right) z^{2} K_{2}(z)
$$

with $g_{X}$ being the number of the degrees of freedom for the particle $X$. We assume that the SM particles are always in the thermal bath and neglect the inverse decay $N_{1} \chi \rightarrow N_{2}$ because the contribution from this process is small.

Using eqs. (3.5)-(3.7), we obtain

$$
\begin{aligned}
Y_{\chi}(\infty) & =\int_{x_{I}}^{\infty} d x \frac{d Y_{\chi}(x)}{d x} \\
& =\frac{\Gamma_{N_{2} \rightarrow N_{1} \chi} \Gamma_{N_{2} \rightarrow \mathrm{B}}}{\Gamma_{N_{2} \rightarrow N_{1} \chi}+\Gamma_{N_{2} \rightarrow \mathrm{B}}} \int_{x_{I}}^{\infty} d x \frac{1}{H x} \frac{K_{1}\left(r_{2} x\right)}{K_{2}\left(r_{2} x\right)} Y_{N_{2}}^{\mathrm{eq}}\left(r_{2} x\right),
\end{aligned}
$$

where we have assumed $Y_{N_{2}}\left(x_{I}\right)=Y_{N_{2}}(\infty)=0$. The integral eq. (3.9) can be carried out approximately and the Majoron relic abundance is evaluated as

$$
\begin{aligned}
Y_{\chi}(\infty) & \approx\left(\frac{\Gamma_{N_{2} \rightarrow N_{1}} \Gamma_{N_{2} \rightarrow \mathrm{B}}}{\Gamma_{N_{2} \rightarrow N_{1} \chi}+\Gamma_{N_{2} \rightarrow \mathrm{B}}}\right) \frac{405 \sqrt{5}}{8 \pi^{9 / 2} g_{*}^{S} g_{*}^{1 / 2}} \frac{m_{\mathrm{Pl}}}{M_{N_{2}}^{2}} \\
& \approx \Gamma_{N_{2} \rightarrow N_{1} \chi} \frac{405 \sqrt{5}}{8 \pi^{9 / 2} g_{*}^{S} g_{*}^{1 / 2}} \frac{m_{\mathrm{Pl}}}{M_{N_{2}}^{2}},
\end{aligned}
$$

where we have used $\Gamma_{N_{2} \rightarrow N_{1} \chi} \ll \Gamma_{N_{2} \rightarrow \mathrm{B}}$ and the explicit expressions of the Hubble parameter and the entropy density.

The time evolution of the yields are shown in figure 3. The masses for the particle contents are fixed as $M_{N_{1}}=5 \mathrm{TeV}, M_{N_{2}}=20 \mathrm{TeV}$ and $m_{\chi}=1 \mathrm{TeV}$, and the off-diagonal 

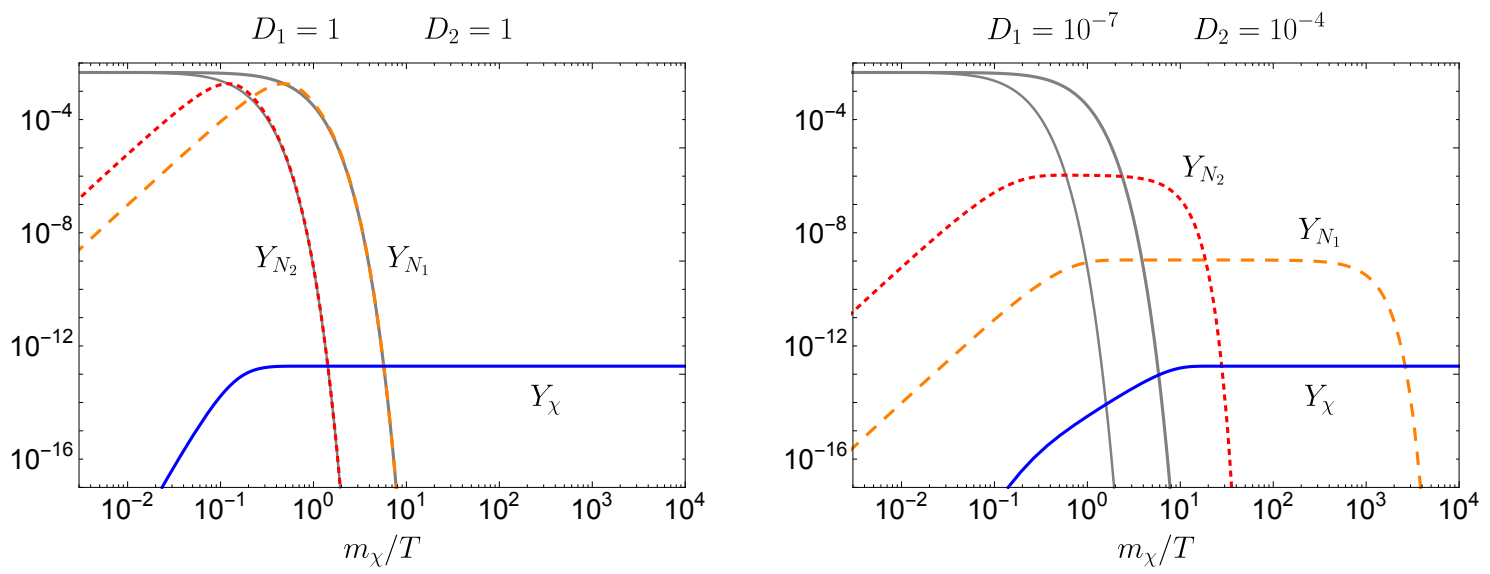

Figure 3. The solutions of the Boltzmann equations with the masses $M_{N_{1}}=5 \mathrm{TeV}, M_{N_{2}}=20 \mathrm{TeV}$, $m_{\chi}=1 \mathrm{TeV}$ and the Yukawa coupling $g=10^{-11}$. The black solid lines indicate the yields in the thermal equilibrium, $Y_{N_{i}}^{\text {eq }}$. The decay parameters $D_{i}=\Gamma_{N_{i} \rightarrow \mathrm{B}} / H\left(T=M_{N_{i}}\right)$ are changed. In the left panel, the decay parameters are unity. In the right panel, the decay parameters are hierarchical and tiny values.

Yukawa coupling is chosen as $g=10^{-11}$. The decay parameter $D_{i}$ is defined by the ratio of the decay width of $N_{i} \rightarrow \mathrm{SM}$ to the Hubble parameter $H$ as $D_{i}=\Gamma_{N_{i} \rightarrow \mathrm{B}} / H\left(T=M_{N_{i}}\right)$ and is related to the neutrino Yukawa couplings $Y_{\alpha i}^{\nu}$ (see eq. (3.4)). In the left panel, the two decay parameters are unity, and then the RH neutrinos go into the thermal bath and the yields follow the thermal equilibrium distribution (black solid lines in the figure). In the right panel, the two decay parameters are too small to put $Y_{N_{i}}$ into the thermal bath. It is interesting that the final result $Y_{\chi}(\infty)$ converges to the same value independently of the magnitudes of the neutrino Yukawa couplings $Y_{\alpha i}^{\nu}$, which is clear from eq. (3.11). This is because, for small $Y_{\alpha i}^{\nu}$, the thermally induced amount of the $\mathrm{RH}$ neutrinos around their mass scale becomes small while the branching ratio decaying into the Majoron becomes large and these two effects are canceled out. In the thermal historical point of view, the independence of neutrino Yukawa couplings is understood by the fact that thermally induced $N_{2}$ is proportional to $D_{2}$ and the time interval where the decay $N_{2} \rightarrow N_{1} \chi$ is effective is inversely proportional to $D_{2}$.

Then the relic abundance of the Majoron is given by

$$
\begin{aligned}
\Omega_{\chi} h^{2} & =\frac{m_{\chi} Y_{\chi}(\infty) s_{0}}{\varepsilon_{c, 0} / h^{2}} \\
& \approx 0.1075 \times\left(\frac{g}{10^{-11}}\right)^{2}\left(\frac{100}{g_{*}^{S}}\right)\left(\frac{100}{g_{*}}\right)^{1 / 2}\left(\frac{m_{\chi}}{1 \mathrm{TeV}}\right)\left(\frac{20 \mathrm{TeV}}{M_{N_{2}}}\right) I\left(\frac{M_{N_{1}}}{M_{N_{2}}}, \frac{m_{\chi}}{M_{N_{2}}}\right),
\end{aligned}
$$

where $s_{0}=2891 \mathrm{~cm}^{-3}$ is the today entropy density, and $\varepsilon_{c, 0}=5.16(h / 0.7)^{2} \mathrm{GeV} \mathrm{m}^{-3}$ is the today critical energy density. The current observed value of DM abundance is $\Omega_{\mathrm{DM}} h^{2}=0.1200(12)[4]$. As we stated above, the relic abundance is independent of the neutrino Yukawa couplings $Y_{\alpha i}^{\nu}$. 

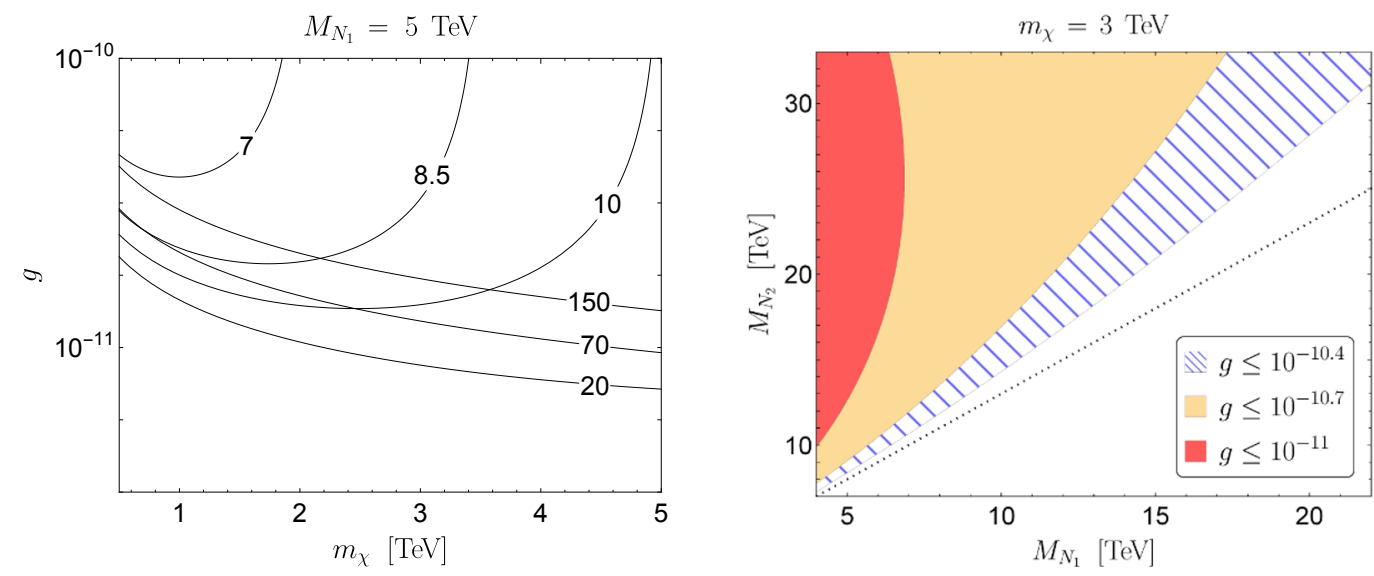

Figure 4. The allowed region to realize the DM relic abundance in $\left(m_{\chi}, g\right)$ - and $\left(M_{N_{1}}, M_{N_{2}}\right)$-planes. See the text for details.

In figure 4 , we show the allowed parameter regions in $\left(m_{\chi}, g\right)$ and $\left(M_{N_{1}}, M_{N_{2}}\right)$ planes. In the left panel, each line represents the parameter space realizing the DM relic abundance for several choices of $M_{N_{2}}$ with $M_{N_{1}}$ fixed as $5 \mathrm{TeV}$. Note that the stronger Yukawa coupling $g$ is required for the smaller Majorana mass $M_{N_{2}}$ since the phase factor $I\left(M_{N_{1}} / M_{N_{2}}, m_{\chi} / M_{N_{2}}\right)$ becomes smaller. On the other hand, large $g$ is also required for larger $M_{N_{2}}$ because $I \sim 1$ and the relic abundance is inversely proportional to $M_{N_{2}}$. The allowed region regarding $M_{N_{2}}$ as a free parameter is bounded from below by the critical line corresponding to $M_{N_{2}} \sim 20 \mathrm{TeV}$. Thus the lower bound for $g$ is around $g \sim 10^{-11}$. In the right panel, we show the allowed region with the DM mass $m_{\chi}=3 \mathrm{TeV}$ and the Yukawa coupling $g \leq 10^{-10.4}, 10^{-10.7}, 10^{-11}$. If we take a severer bound for the offdiagonal Yukawa coupling $g \leq 10^{-11}$ (red region in the figure), the lightest RH neutrino mass has to be in $3 \mathrm{TeV} \leq M_{N_{1}} \leq 7 \mathrm{TeV}$, and the mass $M_{N_{2}}$ has to be larger than $10 \mathrm{TeV}$.

Interestingly, the bound on $g$ for this scenario to work is marginally comparable with the experimentally constrained upper bound eq. (3.2). Therefore, the scenario (A) can be proved or excluded in the near future observations.

\section{3 (B): scalar portal interaction}

Let us move to another scenario, in which we introduce the mixing coupling $\lambda_{H \Phi}|H|^{2}|\Phi|^{2}$. We consider the freeze-in creation of the Majoron in this model. The scalar potential is written as

$$
V(H, \Phi)=V_{H}(H)-\frac{\mu_{\Phi}^{2}}{2}|\Phi|^{2}+\frac{\lambda_{\Phi}}{2}|\Phi|^{4}+\lambda_{H \Phi}|H|^{2}|\Phi|^{2}-\frac{m^{2}}{4}\left(\Phi^{2}+\Phi^{* 2}\right),
$$

and the conditions for the quartic couplings such that the potential is bounded from below are $\lambda_{H}>0, \lambda_{\Phi}>0, \sqrt{\lambda_{H} \lambda_{\Phi}}+\lambda_{H \Phi}>0$. In addition, the quartic coupling $\lambda_{\Phi}$ has the upper bound $8 \pi / 3$ from the perturbative unitarity as discussed in ref. [44]. The quartic coupling $\lambda_{H \Phi}$ is also constrained by the bound of the mixing angle between the CP-even components [45]. 

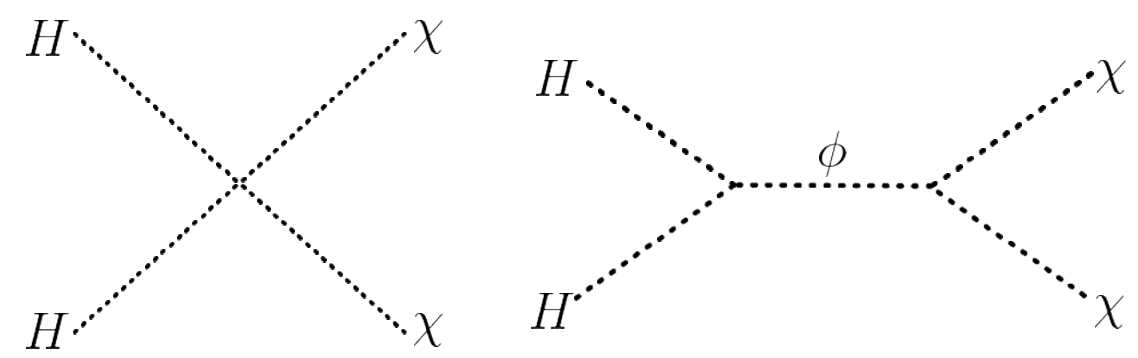

Figure 5. The Feynman diagrams for the Majorogenesis in the scenario (B). The contact type interaction in the left panel is canceled by the low-energy contribution from the scalar mediated interaction in the right panel.

One important feature of the Majoron is a cancellation due to the nature of NGB in two-body scattering processes such as figure 5. The contribution from the contact type four-point interaction (left panel) is canceled by the one from the $\phi$-mediated interaction (right panel) in the soft limit, and the remaining value is suppressed by the large decay constant. Indeed, the leading contribution after the cancellation comes from the portal energy in the propagator, which is written as

$$
i \mathcal{M}\left(H^{\dagger} H \rightarrow \chi \chi\right)(s)=-i \frac{\lambda_{H \Phi}}{s-m_{\phi}^{2}} s
$$

where $s$ is the Mandelstam's $s$ variable and $m_{\phi}^{2}=\lambda_{\Phi} v_{\phi}^{2}$ is the mass of $\phi$. This is consistent with the result implied by the soft-pion theorem, and is easily understood in the non-linear representation:

$$
\Phi=\frac{v_{\phi}+\phi}{\sqrt{2}} e^{i \pi / v_{\phi}}
$$

The phase field $\pi$ is the Majoron in this representation and is the same as $\chi$ to the leading order of $1 / v_{\phi}$. We have the following interaction vertices in the Lagrangian:

$$
\mathcal{L}_{\text {int }} \supset \frac{\phi}{v_{\phi}}\left[\left(\partial_{\mu} \pi\right)^{2}-m_{\chi}^{2} \pi^{2}\right]-\lambda_{H \Phi} v_{\phi} \phi|H|^{2},
$$

where the derivative coupling between the (p)NGB $\pi$ and the CP-even scalar particle $\phi$ has come from the kinetic term of $\Phi$. The scattering amplitude for $H H \rightarrow \pi \pi$ evaluated from this interaction Lagrangian eq. (3.16) is the same as eq. (3.14), which is now given by a single diagram like the right-panel of figure 5 and the energy $(s)$ dependence originates from the derivative coupling.

The Boltzmann equation for the Majoron DM is given by

$$
\frac{d Y_{\chi}(x)}{d x}=\frac{2}{s H x} \gamma^{\chi \chi}{ }_{H^{\dagger} H},
$$

where $\gamma^{\chi \chi}{ }_{H^{\dagger} H}$ is the interaction density defined as

$$
\gamma_{H^{\dagger} H}^{\chi \chi} \equiv \frac{4}{2 ! 2 !} \frac{T \lambda_{H \Phi}^{2}}{2^{9} \pi^{5}} \int_{4 m_{\chi}^{2}}^{\infty} d s \sqrt{s-4 m_{\chi}^{2}} K_{1}(\sqrt{s} / T) \frac{s^{2}}{\left(s-m_{\phi}^{2}\right)^{2}} .
$$




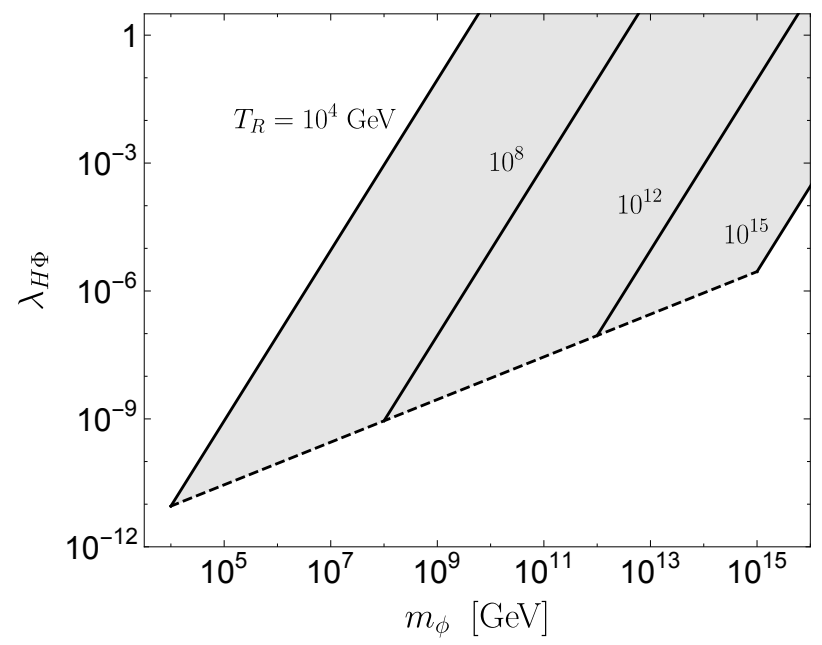

Figure 6. The parameter space in the $\left(m_{\phi}, \lambda_{H \Phi}\right)$ plane explaining the DM relic by the TeV scale Majoron. See the text for details.

The prefactor 4 comes from the degrees of freedom of the Higgs doublet in the symmetric phase. We integrate the Boltzmann equation with the initial condition $Y_{\chi}\left(x_{R}\right)=0$, then the Majoron abundance can be analytically evaluated as

$$
\Omega_{\chi} h^{2} \approx 1.5 \times 10^{25} \mathrm{GeV}\left(\frac{100}{g_{*}^{S}}\right)\left(\frac{100}{g_{*}}\right)^{1 / 2}\left(\frac{m_{\chi}}{1 \mathrm{TeV}}\right) \frac{\lambda_{H \Phi}^{2} T_{R}^{3}}{m_{\phi}^{4}},
$$

where $T_{R}$ is the reheating temperature satisfying $x_{R} \equiv m_{\chi} / T_{R}$. In eq. (3.19), we have assumed that $m_{\phi}$ is larger than the reheating temperature. Due to the energy dependence of the amplitude (3.14) and the heavy portal scalar, the relic abundance is dominated by the contribution from the ultraviolet(UV)-region unlike the previous case (A) and depends on the reheating temperature $T_{R}$, which arises from the UV physics. A similar type of freeze-in effect is discussed in the context of higher dimensional operators [41].

In figure 6 , we show the allowed region in the $\left(m_{\phi}, \lambda_{H \Phi}\right)$ plane. Each solid line represents the parameter space realizing the DM relic abundance and the region above each line for $T_{R}$ being fixed is excluded by the over creation. The dashed line means the case $m_{\phi}=T_{R}$, and the region below this line is not valid because we assumed that the mass of $\phi$ is larger than the reheating temperature such that $\phi$ is inactive in thermal evolution after the reheating. The shaded region shows the parameter space in which the DM relic abundance is realized regarding the reheating temperature as a free parameter. The region of $m_{\phi}$ is taken as $10^{4} \mathrm{GeV} \leq m_{\phi} \leq 10^{15} \mathrm{GeV}$. We note the VEV $v_{\phi}$ is large for the $\mathrm{TeV}$-scale Majoron and the constraint from the mixing among the CP-even components is negligible due to the suppression by $v_{\phi}$.

We here give a comment on other previous work. The portal-like coupling of pNGB has also been discussed in various contexts, e.g., refs. [18, 31]. The existence of the contact type interaction by the quartic coupling $\lambda_{H \Phi}$ is usually assumed, but that is canceled by heavy scalar mediated contribution, as stated above. Consequently, it seems that the 

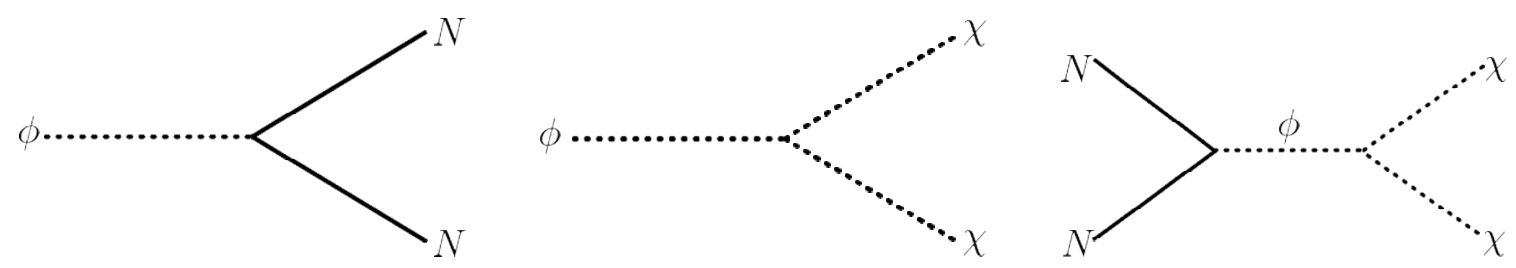

Figure 7. The Feynman diagrams for the Majorogenesis in the scenario (C).

thermal freeze-out creation and collider search of the pNGB DM are inaccessible in case of the large decay constant.

\section{4 (C): resonant creation from non-thermal source}

Let us consider the third scenario that the Majoron DM is created by the $\mathrm{RH}$ neutrino annihilation process mediated by the heavy CP-even scalar $\phi$. We here assume that the mass $m_{\phi}$ is smaller than the reheating temperature $T_{R}$ so that $\phi$ plays an important role in the thermal history of the universe. In this subsection, we consider the case of one generation $\mathrm{RH}$ neutrino for simplicity, but the generalization to three generations $\mathrm{RH}$ neutrino is straightforward.

We further assume that the RH neutrino has a Yukawa coupling to the inflaton field $\varphi$ with mass $m_{\varphi}$. This coupling generates the $\mathrm{RH}$ neutrinos non-thermally during the reheating, and the yield at $T_{R}$ is evaluated as

$$
Y_{N}=\frac{3}{2} \frac{T_{R}}{m_{\varphi}} \operatorname{Br}(\varphi \rightarrow N N)
$$

Here $\operatorname{Br}(\varphi \rightarrow N N)$ is the branching ratio of $\varphi \rightarrow N N$ process, which is given by $\Gamma_{\varphi \rightarrow N N} / \Gamma_{\varphi}$ with $\Gamma_{\varphi}$ being the total decay width of the inflaton. The reheating temperature $T_{R}$ is defined by $H\left(T=T_{R}\right)=\Gamma_{\varphi}$.

The $\mathrm{RH}$ neutrinos created by the inflaton can annihilate into the Majoron through the scattering process mediated by $\phi: N N \stackrel{\phi}{\longleftrightarrow} \chi \chi$ as shown by figure 7 . The Yukawa coupling $f$ corresponding to $\phi \bar{N} N$ should be small from astrophysical constraints, and the three point coupling $\phi \chi \chi$ is also suppressed. As we will see in the following, even for these tiny couplings, a sufficient amount of the Majoron DM can be generated with the resonant contribution of $\phi$. The partial decay widths of $\phi$ to RH neutrinos and Majoron

$$
\Gamma_{\phi \rightarrow N N}=\frac{f^{2} m_{\phi}}{32 \pi}\left[1-\frac{4 M_{N}^{2}}{m_{\phi}^{2}}\right]^{3 / 2}, \quad \Gamma_{\phi \rightarrow \chi \chi}=\frac{\lambda_{\Phi} m_{\phi}}{32 \pi}\left[1-\frac{4 m_{\chi}^{2}}{m_{\phi}^{2}}\right]^{1 / 2} .
$$

The contribution to the Boltzmann equations from the $\phi$ portal annihilation process, $N N \stackrel{\phi}{\rightarrow} \chi \chi$, is evaluated as

$$
\begin{aligned}
\gamma^{N N}{ }_{\chi \chi} & =\frac{\lambda_{\Phi} f^{2} T}{2^{11} \pi^{5}} \int_{4 M_{N}^{2}}^{\infty} d s \frac{\left(s-4 M_{N}^{2}\right)^{3 / 2}\left(s-4 m_{\chi}^{2}\right)^{1 / 2}}{s^{1 / 2}\left(s-m_{\phi}^{2}\right)^{2}} K_{1}(\sqrt{s} / T) \\
& \approx \frac{f^{2} m_{\phi}^{3} T}{2^{6} \pi^{3}}\left[1-\frac{4 M_{N}^{2}}{m_{\phi}^{2}}\right]^{3 / 2} K_{1}\left(m_{\phi} / T\right),
\end{aligned}
$$

where we use the narrow width approximation. 
The Boltzmann equations for the Majorogenesis in this system are expressed as

$$
\begin{aligned}
\frac{d Y_{N}(x)}{d x}= & 2 \frac{\Gamma_{\phi \rightarrow N N}}{H x} \frac{K_{1}\left(r_{\phi} x\right)}{K_{2}\left(r_{\phi} x\right)} Y_{\phi}^{\mathrm{eq}}\left(r_{\phi} x\right)\left[\frac{Y_{\phi}(x)}{Y_{\phi}^{\mathrm{eq}}\left(r_{\phi} x\right)}-\left(\frac{Y_{N}(x)}{Y_{N}^{\mathrm{eq}}(r x)}\right)^{2}\right] \\
& -\frac{2}{H s x} \gamma^{N N} \chi \chi\left(\frac{Y_{N}(x)}{Y_{N}^{\mathrm{eq}}(r x)}\right)^{2}-\frac{\Gamma_{N \rightarrow \mathrm{B}}}{H x} \frac{K_{1}(r x)}{K_{2}(r x)}\left[Y_{N}(x)-Y_{N}^{\mathrm{eq}}(r x)\right], \\
\frac{d Y_{\phi}(x)}{d x}= & -\frac{\Gamma_{\phi \rightarrow N N}}{H x} \frac{K_{1}\left(r_{\phi} x\right)}{K_{2}\left(r_{\phi} x\right)} Y_{\phi}^{\mathrm{eq}}\left(r_{\phi} x\right)\left[\frac{Y_{\phi}(x)}{Y_{\phi}^{\mathrm{eq}}\left(r_{\phi} x\right)}-\left(\frac{Y_{N}(x)}{Y_{N}^{\mathrm{eq}}(r x)}\right)^{2}\right] \\
& -\frac{\Gamma_{\phi \rightarrow \chi \chi}}{H x} \frac{K_{1}\left(r_{\phi} x\right)}{K_{2}\left(r_{\phi} x\right)} Y_{\phi}(x), \\
\frac{d Y_{\chi}(x)}{d x}= & 2 \frac{\Gamma_{\phi \rightarrow \chi \chi}}{H x} \frac{K_{1}\left(r_{\phi} x\right)}{K_{2}\left(r_{\phi} x\right)} Y_{\phi}(x)+\frac{2}{H s x} \gamma^{N N}{ }_{\chi \chi}\left(\frac{Y_{N}(x)}{Y_{N}^{\mathrm{eq}}(r x)}\right)^{2}
\end{aligned}
$$

where we have assumed that the SM particles are in the thermal bath. $r_{\phi}$ and $r$ are defined as $r_{\phi} \equiv m_{\phi} / m_{\chi}$ and $r \equiv M_{N} / m_{\chi}$, respectively. The relic density of the Majoron DM is found by solving these equations and evaluated approximately as

$$
Y_{\chi}(\infty) \approx \frac{f^{2} m_{\phi}}{16 \pi}\left(1-\frac{4 M_{N}^{2}}{m_{\phi}^{2}}\right)^{3 / 2} \int_{x_{R}}^{\infty} d x \frac{2}{H x} \frac{K_{1}\left(r_{\phi} x\right)}{K_{2}\left(r_{\phi} x\right)} Y_{\phi}^{\mathrm{eq}}\left(r_{\phi} x\right)\left(\frac{Y_{N}(x)}{Y_{N}^{\mathrm{eq}}(r x)}\right)^{2},
$$

where we have used the boundary conditions $Y_{\phi}\left(x_{R}\right)=Y_{\chi}\left(x_{R}\right)=Y_{N}(\infty)=Y_{\phi}(\infty)=0$. The final result is given by

$$
Y_{\chi}(\infty) \approx \frac{\pi^{5 / 2} g_{*}^{S}}{128 \sqrt{5} g_{*}^{1 / 2}} \frac{m_{\mathrm{Pl}}}{m_{\phi}} f^{2} Y_{N}\left(x_{R}\right)^{2}
$$

The relic abundance of the Majoron DM is evaluated as

$$
\Omega_{\chi} h^{2} \approx 4.02 \times 10^{27}\left(\frac{100}{g_{*}^{S}}\right)\left(\frac{100}{g_{*}}\right)^{1 / 2} \frac{m_{\chi}}{m_{\phi}} f^{2} Y_{N}\left(x_{R}\right)^{2} .
$$

This result depends on the Yukawa coupling $f$, the scalar mass $m_{\phi}$, and the initial amount of RH neutrinos, but is independent of the RH neutrino mass.

The time evolution of the yields are shown in figure 8, in which the masses are fixed as $m_{\phi}=10^{6} \mathrm{GeV}, m_{\chi}=10^{3} \mathrm{GeV}$ and $M_{N}=10^{4} \mathrm{GeV}$. The yield $Y_{N}$ initially created by the inflaton decay is large and remains the constant for $T \gtrsim m_{\phi}$, during which $\phi$ and Majoron are generated through the decay and scattering processes. After the creation of Majoron DM by this process, the relic abundance is frozen-in at the temperature just below $m_{\phi}{ }^{3}$ On the other hand, the heavy scalar $\phi$ similarly created by the $N$ decay finally disappears after the $\phi \rightarrow \chi \chi$ process becomes effective in the thermal history.

In figure 9 , we show the allowed region in $\left(m_{\chi}, m_{\phi}\right)$ plane with the initial yield $f Y_{N}\left(x_{R}\right) \geq 10^{-11}, 10^{-12}, 10^{-13}$. A smaller $\phi$ mass is favored to realize the DM relic abundance. The figure shows that a tiny value of the coupling $f$ is compatible with the observations, while that depends on the other parameters.

\footnotetext{
${ }^{3}$ The relic abundance of the Majoron could be slightly changed by thermalized RH neutrinos. However, it is not large effect unless $m_{\phi}$ is close to $M_{N}$.
} 


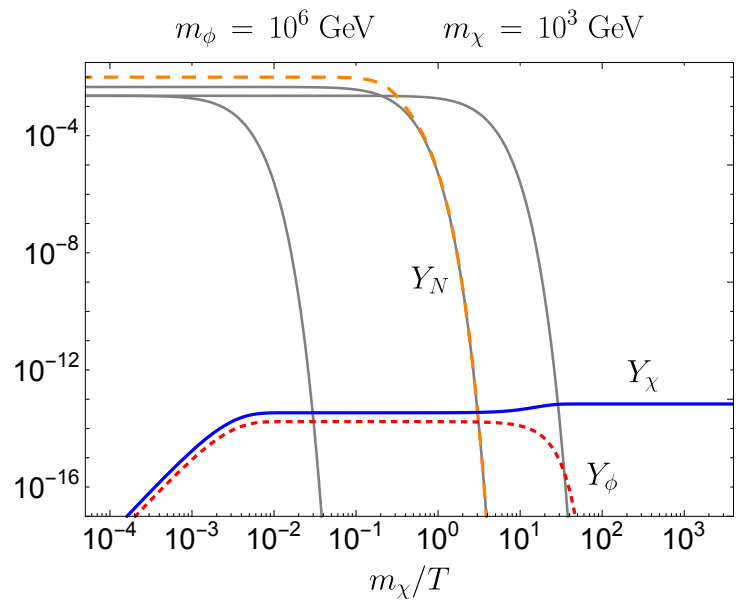

Figure 8. The solution of the Boltzmann equations with $m_{\phi}=10^{6} \mathrm{GeV}, m_{\chi}=10^{3} \mathrm{GeV}$ and the initial value of the RH neutrino yield $Y_{N}\left(x_{R}\right)=10^{-2}$. The RH neutrino mass is fixed as $M_{N}=10^{4} \mathrm{GeV}$, but the DM abundance $Y_{\chi}(\infty)$ does not depend on it.

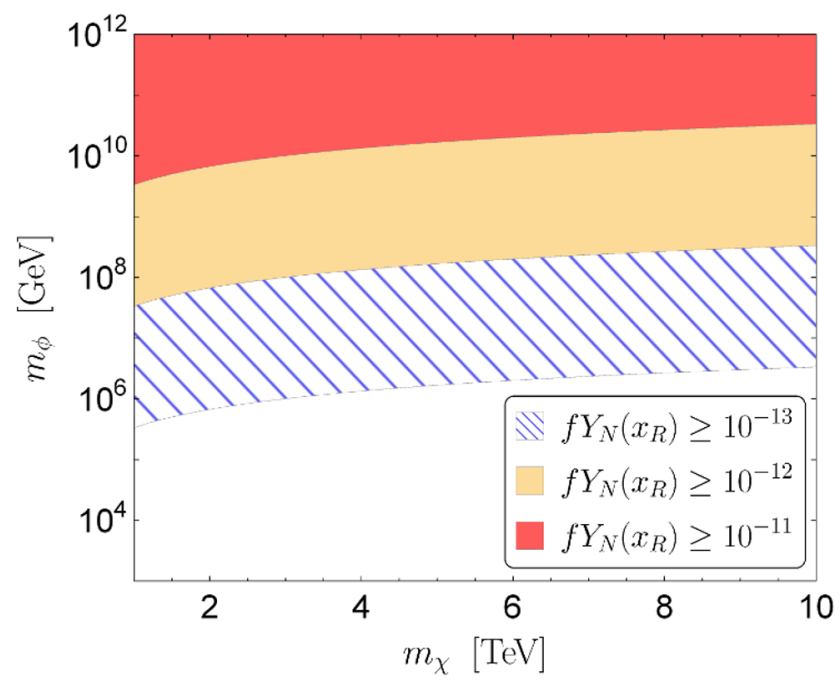

Figure 9. The allowed region in $\left(m_{\chi}, m_{\phi}\right)$ plane when the initial yield of the RH neutrino is given. The initial yield is fixed as $f Y_{N}\left(x_{R}\right) \geq 10^{-13}, 10^{-12}, 10^{-11}$, and the small $\phi$ mass is favored.

\section{Summary and outlook}

We have studied the scenarios where the Majoron, a pNGB of lepton number symmetry with $\mathrm{TeV}$-scale mass, can be the DM of the universe. Since the decay constant of the Majoron is large and the coupling to the SM is tiny, it is nontrivial how to create the Majoron in the early universe, called Majorogenesis. The Majoron model can realize neither freeze-out nor freeze-in production of the Majoron DM with the large VEV because the Majoron couplings to the SM particles are tiny and the Yukawa couplings to the RH neutrinos are flavor-diagonal in the mass basis of the $\mathrm{RH}$ neutrinos. To avoid this flaw, we have discussed three scenarios $(\mathrm{A})-(\mathrm{C})$ for Majorogenesis via the freeze-in mechanism; 
(A) introducing explicit Majorana masses, (B) using the interaction with the SM Higgs doublet, (C) using the resonant production from the non-thermally induced $\mathrm{RH}$ neutrinos.

In (A), we find the lower bound on the Majoron Yukawa coupling for the freezein Majorogenesis to work, and the bound is roughly comparable with the tiny value of Yukawa coupling constrained from astrophysics. Therefore, this scenario could be proved or excluded in the near future observations such as Cherenkov Telescope Array (CTA) [46] and IceCube Neutrino Observatory [47].

In (B), the toal coupling between the Majoron and the SM Higgs is found to be canceled and suppressed by the large mass scale, and is useful to create the Majoron via the freeze-in mechanism. Note that this scenario is quite general because we have used only the fact that $\chi$ is the pNGB having the large VEV and the mixing coupling to the SM Higgs.

In (C), the sufficient amount of RH neutrinos are produced by the decay of the inflaton during the reheating. After that, the $\phi$-mediated $N N \chi \chi$ interaction, whose magnitude is constrained by cosmic-ray observations, can be used to realize the freeze-in production.

In all the scenarios $(\mathrm{A})-(\mathrm{C})$, there are the parameter regions realizing the DM relic abundance and avoiding the astrophysical constraints. Therefore, the Majoron with the $\mathrm{TeV}$-scale mass (or heavier) can play the role of DM in the universe.

For further study, it may be intersting to examine the leptogenesis [48] in these scenarios. A straightforward way is using resonances between the $\mathrm{RH}$ neutrinos [49]. A more challenging is introducing other particles whose masses are at an intermediate scale between $v$ and $v_{\phi}$. One can use radiative decay processes of $\mathrm{RH}$ neutrinos where the new particles appear in the loop to generate lepton asymmetry. This motivates us to consider an extension in which one more SM-singlet $\mathrm{U}(1)_{L}$-charged scalar is added. Whether such type of leptogenesis can be compatible with the $\mathrm{TeV}$-scale Majorogeneis is left for future work [50].

\section{Acknowledgments}

The authors thank Motoko Fujiwara and Takashi Toma for useful discussions and comments. The work is supported by JSPS Grant-in-Aid for Scientific Research, No. JP20J11901 (Y.A.), No. JP18J22733 (Y.H.) and No. JP18H01214 (K.Y.).

Open Access. This article is distributed under the terms of the Creative Commons Attribution License (CC-BY 4.0), which permits any use, distribution and reproduction in any medium, provided the original author(s) and source are credited.

\section{References}

[1] E. Corbelli and P. Salucci, The Extended Rotation Curve and the Dark Matter Halo of M33, Mon. Not. Roy. Astron. Soc. 311 (2000) 441 [astro-ph/9909252] [INSPIRE].

[2] Y. Sofue and V. Rubin, Rotation curves of spiral galaxies, Ann. Rev. Astron. Astrophys. 39 (2001) 137 [astro-ph/0010594] [INSPIRE].

[3] R. Massey, T. Kitching and J. Richard, The dark matter of gravitational lensing, Rept. Prog. Phys. 73 (2010) 086901 [arXiv: 1001.1739] [INSPIRE]. 
[4] Planck collaboration, Planck 2018 results. VI. Cosmological parameters, arXiv: 1807.06209 [INSPIRE].

[5] S.W. Randall, M. Markevitch, D. Clowe, A.H. Gonzalez and M. Bradac, Constraints on the Self-Interaction Cross-Section of Dark Matter from Numerical Simulations of the Merging Galaxy Cluster 1E 0657-56, Astrophys. J. 679 (2008) 1173 [arXiv:0704.0261] [INSPIRE].

[6] Fermi-LAT collaboration, Dark Matter Constraints from Observations of 25 Milky Way Satellite Galaxies with the Fermi Large Area Telescope, Phys. Rev. D 89 (2014) 042001 [arXiv: 1310.0828] [INSPIRE].

[7] AMS collaboration, Dark matter searches with AMS-02, PoS HEP2005 (2006) 005 [INSPIRE].

[8] LUX collaboration, Limits on spin-dependent WIMP-nucleon cross section obtained from the complete LUX exposure, Phys. Rev. Lett. 118 (2017) 251302 [arXiv:1705.03380] [InSPIRE].

[9] PandaX-II collaboration, Dark Matter Results From 54-Ton-Day Exposure of PandaX-II Experiment, Phys. Rev. Lett. 119 (2017) 181302 [arXiv:1708.06917] [INSPIRE].

[10] XENON collaboration, Dark Matter Search Results from a One Ton-Year Exposure of XENON1T, Phys. Rev. Lett. 121 (2018) 111302 [arXiv:1805.12562] [INSPIRE].

[11] Particle Data Group collaboration, Review of Particle Physics, Phys. Rev. D 98 (2018) 030001 [INSPIRE].

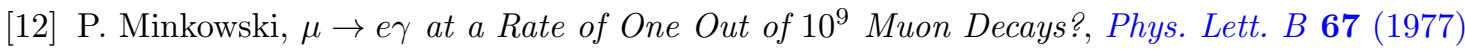
421 [INSPIRE].

[13] T. Yanagida, Horizontal Symmetry and Masses of Neutrinos, Prog. Theor. Phys. 64 (1980) 1103 [INSPIRE].

[14] M. Gell-Mann, P. Ramond and R. Slansky, Complex Spinors and Unified Theories, Conf. Proc. C $\mathbf{7 9 0 9 2 7}$ (1979) 315 [arXiv: 1306.4669] [INSPIRE].

[15] Y. Chikashige, R.N. Mohapatra and R.D. Peccei, Spontaneously Broken Lepton Number and Cosmological Constraints on the Neutrino Mass Spectrum, Phys. Rev. Lett. 45 (1980) 1926 [INSPIRE].

[16] Y. Chikashige, R.N. Mohapatra and R.D. Peccei, Are There Real Goldstone Bosons Associated with Broken Lepton Number?, Phys. Lett. B 98 (1981) 265 [INSPIRE].

[17] G.B. Gelmini and M. Roncadelli, Left-Handed Neutrino Mass Scale and Spontaneously Broken Lepton Number, Phys. Lett. B 99 (1981) 411 [INSPIRE].

[18] P.-H. Gu, E. Ma and U. Sarkar, Pseudo-Majoron as Dark Matter, Phys. Lett. B 690 (2010) 145 [arXiv: 1004.1919] [INSPIRE].

[19] F. Bazzocchi, M. Lattanzi, S. Riemer-Sørensen and J.W.F. Valle, X-ray photons from late-decaying majoron dark matter, JCAP 08 (2008) 013 [arXiv:0805.2372] [INSPIRE].

[20] V. Berezinsky and J.W.F. Valle, The KeV majoron as a dark matter particle, Phys. Lett. B 318 (1993) 360 [hep-ph/9309214] [INSPIRE].

[21] M. Lattanzi, S. Riemer-Sorensen, M. Tortola and J.W.F. Valle, Updated CMB and $x$ - and $\gamma$-ray constraints on Majoron dark matter, Phys. Rev. D 88 (2013) 063528 [arXiv: 1303.4685] [INSPIRE].

[22] G. Gelmini, D.N. Schramm and J.W.F. Valle, Majorons: A Simultaneous Solution to the Large and Small Scale Dark Matter Problems, Phys. Lett. B 146 (1984) 311 [INSPIRE]. 
[23] J. Heeck and H.H. Patel, Majoron at two loops, Phys. Rev. D 100 (2019) 095015 [arXiv: 1909.02029] [INSPIRE].

[24] V. Barger, M. McCaskey and G. Shaughnessy, Complex Scalar Dark Matter vis-à-vis CoGeNT, DAMA/LIBRA and XENON100, Phys. Rev. D 82 (2010) 035019 [arXiv: 1005.3328] [INSPIRE].

[25] C. Gross, O. Lebedev and T. Toma, Cancellation Mechanism for Dark-Matter-Nucleon Interaction, Phys. Rev. Lett. 119 (2017) 191801 [arXiv:1708.02253] [INSPIRE].

[26] D. Azevedo, M. Duch, B. Grzadkowski, D. Huang, M. Iglicki and R. Santos, One-loop contribution to dark-matter-nucleon scattering in the pseudo-scalar dark matter model, JHEP 01 (2019) 138 [arXiv:1810.06105] [INSPIRE].

[27] K. Ishiwata and T. Toma, Probing pseudo Nambu-Goldstone boson dark matter at loop level, JHEP 12 (2018) 089 [arXiv: 1810.08139] [INSPIRE].

[28] S. Palomares-Ruiz, Model-Independent Bound on the Dark Matter Lifetime, Phys. Lett. B 665 (2008) 50 [arXiv:0712.1937] [INSPIRE].

[29] L. Covi, M. Grefe, A. Ibarra and D. Tran, Neutrino Signals from Dark Matter Decay, JCAP 04 (2010) 017 [arXiv: 0912.3521] [INSPIRE].

[30] S. Matsumoto and K. Yoshioka, Deep Correlation Between Cosmic-Ray Anomaly and Neutrino Masses, Phys. Rev. D 82 (2010) 053009 [arXiv:1006.1688] [INSPIRE].

[31] F.S. Queiroz and K. Sinha, The Poker Face of the Majoron Dark Matter Model: LUX to keV Line, Phys. Lett. B 735 (2014) 69 [arXiv:1404.1400] [INSPIRE].

[32] D. Barducci et al., Monojet searches for momentum-dependent dark matter interactions, JHEP 01 (2017) 078 [arXiv: 1609.07490] [INSPIRE].

[33] K. Huitu, N. Koivunen, O. Lebedev, S. Mondal and T. Toma, Probing pseudo-Goldstone dark matter at the LHC, Phys. Rev. D 100 (2019) 015009 [arXiv: 1812.05952] [INSPIRE].

[34] J.M. Cline and T. Toma, Pseudo-Goldstone dark matter confronts cosmic ray and collider anomalies, Phys. Rev. D 100 (2019) 035023 [arXiv:1906.02175] [INSPIRE].

[35] M. Ruhdorfer, E. Salvioni and A. Weiler, A Global View of the Off-Shell Higgs Portal, SciPost Phys. 8 (2020) 027 [arXiv: 1910.04170] [INSPIRE].

[36] C. Arina, A. Beniwal, C. Degrande, J. Heisig and A. Scaffidi, Global fit of pseudo-Nambu-Goldstone Dark Matter, JHEP 04 (2020) 015 [arXiv:1912.04008] [INSPIRE].

[37] I.Z. Rothstein, K.S. Babu and D. Seckel, Planck scale symmetry breaking and majoron physics, Nucl. Phys. B 403 (1993) 725 [hep-ph/9301213] [INSPIRE].

[38] M. Frigerio, T. Hambye and E. Masso, Sub-GeV dark matter as pseudo-Goldstone from the seesaw scale, Phys. Rev. X 1 (2011) 021026 [arXiv:1107.4564] [InSPIRE].

[39] Y. Abe, T. Toma and K. Tsumura, Pseudo-Nambu-Goldstone dark matter from gauged $\mathrm{U}(1)_{B-L}$ symmetry, JHEP 05 (2020) 057 [arXiv: 2001.03954] [INSPIRE].

[40] N. Okada, D. Raut and Q. Shafi, Pseudo-Goldstone Dark Matter in gauged B - L extended Standard Model, arXiv:2001.05910 [INSPIRE].

[41] L.J. Hall, K. Jedamzik, J. March-Russell and S.M. West, Freeze-In Production of FIMP Dark Matter, JHEP 03 (2010) 080 [arXiv:0911.1120] [INSPIRE]. 
[42] C. Garcia-Cely and J. Heeck, Neutrino Lines from Majoron Dark Matter, JHEP 05 (2017) 102 [arXiv: 1701.07209] [INSPIRE].

[43] R. Iwashima, M. Yamanaka and K. Yoshioka, to appear.

[44] C.-Y. Chen, S. Dawson and I.M. Lewis, Exploring resonant di-Higgs boson production in the Higgs singlet model, Phys. Rev. D 91 (2015) 035015 [arXiv:1410.5488] [InSPIRE].

[45] A. Falkowski, C. Gross and O. Lebedev, A second Higgs from the Higgs portal, JHEP 05 (2015) 057 [arXiv: 1502.01361] [INSPIRE].

[46] CTA collaboration, Prospects for Indirect Dark Matter Searches with the Cherenkov Telescope Array (CTA), PoS ICRC2015 (2016) 1203 [arXiv: 1508.06128] [InSPIRE].

[47] ICECube collaboration, Neutrino astronomy with the next generation IceCube Neutrino Observatory, arXiv: 1911.02561 [INSPIRE].

[48] M. Fukugita and T. Yanagida, Baryogenesis Without Grand Unification, Phys. Lett. B 174 (1986) 45 [INSPIRE].

[49] A. Pilaftsis and T.E.J. Underwood, Resonant leptogenesis, Nucl. Phys. B 692 (2004) 303 [hep-ph/0309342] [INSPIRE].

[50] Y. Abe, Y. Hamada, T. Ohata, K. Suzuki and K. Yoshioka, work in progress. 